\title{
Méthodes d'étude des pluies de courte durée
}

\author{
Investigation methods for short-duration rainfall
}

Jean-Pierre Lahaye*, Maurice Meunier** et Raymond Tartès***

Cette note a été écrite en 1976 pour les Joumées d'étude du groupe Pluviométrie de la SHF. Pour cette publication, il y a simplement eu condensation du texte et des graphiques, mais pas de modification bien que certains points abordés ici soient moins d'actualité en 1979.

\section{Définitions}

Nous nous plaçons ici dans l'optique de respecter deux contraintes qui vont nous permettre de définir notre objet d'étude et de choisir les méthodes d'études appropriées :

- Nous voulons étudier des pluies de courte durée, (quelques jours au maximum) mais uniquement dans le but de calculer des débits.

- Placés dans cette optique pratique, nous voulons garder une rigueur scientifique que l'on perd parfois un peu de vue lorsqu'on ne se soucie que du chiffre à obtenir.

\section{Conséquences dues à la première contrainte}

Le fait essentiel est qu'on n'est pas libre de la définition qu'il faut adopter pour les pluies. Il existe en effet plusieurs définitions possibles. Les plus courantes sont les suivantes (Fig. 1):

1) Pluie de durée fixe, d'origine variable, pluie continue ou discontinue ;

2) Pluie de durée fixe, d'origine variable, pluie continue ;

$\left(^{*}\right)$ Actuellement au CIEH. Haute Volta. En 1976: CTGREF Division Irrigation.

(**) CTGREF - Division Irrigation

(***) SRAE Languedoc-Roussillon.

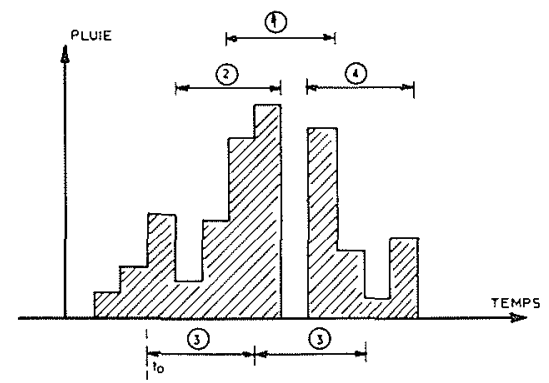

Figure 1 - Exemple de ce qu'on obtient pour les pluies de $4 \mathrm{~h}$ suivant la définition adoptée.

3) Pluie de durée fixe, d'origine fixe, pluie continue ou discontinue ;

4) Pluie totale de l'épisode pluvieux.

Quand on s'intéresse à des débits, on ne peut pas négliger les pluies discontinues car un arrêt bref à l'intérieur d'une averse n'a pas d'effet sur l'hydrogramme. De plus comme les longues pluies continues sont plus rares que les courtes, on peut être conduit à ce paradoxe qu'à fréquence égale, les quantités de pluies tombées en $12 \mathrm{~h}$ (ou $24 \mathrm{~h}$ ) sont inférieures aux quantités de pluies tombées en $1 \mathrm{~h}$ (ou $2 \mathrm{~h}$ ). Ce phénomène est d'ailleurs perceptible avec les célèbres courbes de Grisollet qui ont été établies à partir de la définition $n^{\circ} 2$, puisque la pluie décennale de $5 \mathrm{~h}$ est à peine supérieure à la pluie décennale de $3 \mathrm{~h}(41,8 \mathrm{~mm}$ contre $40,9 \mathrm{~mm})$.

Pour la même raison, on ne peut utiliser la définition $n^{\circ} 4$ des pluies lorsqu'on calcule des débits : en effet, si on génère les débits d'un bassin versant de temps caractéristique $6 \mathrm{~h}$ à partir des pluies de $6 \mathrm{~h}$, et si on utilise la définition $n^{\circ} 4$ des pluies, on admet implicitement que seules les averses de durée totale $6 \mathrm{~h}$ provoquent un écoulement. 


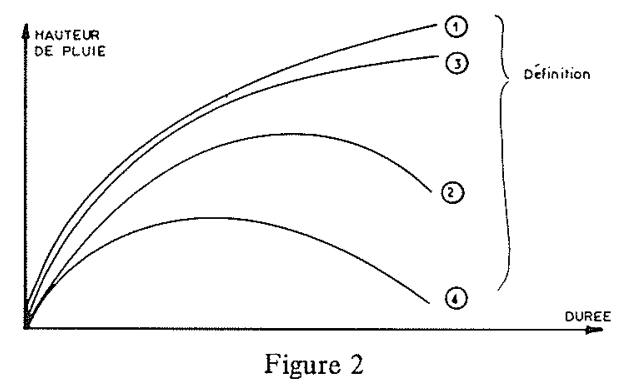

On ne peut donc utiliser que la définition $\mathrm{n}^{\circ} 3$, ou mieux, la définition $n^{\circ} 1$. La définition $n^{\circ} 1$ est celle qui est actuellement adoptée pour les dépouillements de pluviogrammes. La définition $\mathrm{n}^{\circ} 3$ est utilisée lorsqu'on traite de pluies journalières, lorsqu'on utilise la méthode de gradex ou lorsqu'on discrétise les intensités de pluie pour un traitement par ordinateur. L'étude théorique de Weiss (1) confirmée par quelques vérifications expérimentales indique qu'on peut passer de la définition $\mathrm{n}^{\circ} 3$ à la définition $\mathrm{n}^{\circ} 1$, à fréquence égale, en multipliant par le rapport $n / n-0,125$ où $n$ est le nombre de pas de temps de la durée considérée.

La figure 2 montre la forme des courbes des hauteurs de pluie en fonction de leur durée, à fréquence égale, suivant la définition utilisée.

\section{Conséquences dues à la deuxième contrainte}

Conserver un minimum de rigueur scientifique signifie, à notre sens, d'une part ne pas faire d'erreur de raisonnement et, d'autre part faire attention à l'utilisation par d'autres des résultats obtenus.

On peut oublier par exemple de vérifier que deux opérateurs appliqués successivement aux pluies sont commutatifs. Ainsi, dans le calcul des bassins de compensation, le calcul des volumes à stocker et l'étude statistique ne sont pas commutatifs. Il faut les effectuer dans cet ordre. Ce genre d'erreur est facile à faire si on ne réalise pas que les courbes obtenues à partir de données élaborées n'ont pas forcément de signification physique : ainsi dans le cas des bassins de compensation si on effectue l'étude statistique des pluies puis le calcul du volume à stocker, on donne implicitement à la courbe reliant les hauteurs de pluie de fréquence donnée en fonction de la durée, la signification d'une courbe de pluies cumulées, ce qui est évidemment faux. Toutes les courbes intensité, durée, fréquence risquent d'être ainsi interprétées de façon erronée.

Ce type d'erreur est encore facilité par une présentation analytique des résultats élaborés : si l'intensité de la pluie décennale est donnée par $i=a \cdot t^{b}$ ( $t$ durée de la pluie), la quantité de pluie décennale sera donnée par $a . t^{b+1}$; on oublie alors très facilement que, de par sa définition, cette fonction n'est pas continue et qu'on ne peut pas la dériver. Il faut donc penser à l'utilisation qui va être faite des résultats obtenus, car le praticien qui trouve dans une étude que la quantité de pluie décennale

(1) Journal of the Hydraulics-Division, ASCE HY1 Janvier 1964. est donnée par $a \cdot t^{b+1}$ ne se posera pas la question de savoir si cette fonction est continue ou pas. De même il extrapolera plus facilement une fonction analytique qu'une courbe donnée sur un graphique. Bien sûr, on peut dire qu'une relation analytique s'accompagne normalement d'une fourchette de validité. Mais il est si facile de "perdre" cette fourchette quand on passe d'un document à un autre! Il est donc souhaitable de représenter le moins possible les résultats d'une étude sous forme analy tique.

\section{Méthodologie}

Il faut séparer le cas où on envisage une étude complète de la pluviométrie, de celui où l'on se borne à utiliser des résultats existants que l'on critique ou étend.

\section{Etude complète}

\section{Problemes posés}

Le premier problème concerne le dépouillement des pluviogrammes : comment faut-il faire ? Que faut-il tirer comme renseignements supplémentaires qui pourront être utiles par la suite ? Faut-il utiliser un seul pluviographe (le plus proche du lieu d'étude ? la plus longue durée ?) ou bien tous les pluviographes de la région?

Le deuxième problème concerne la variation spatiale des résultats : comment passer des endroits de mesure au site intéressé (où on ne possède en général aucune mesure) ? Y-a-t-il un gradient pluviométrique ?

Le troisième problème concerne la précision de l'ajustement obtenu : comment l'améliorer lorsque les séries de données sont courtes?

Le quatrième problème est en fait conditionné par le troisième ; il concerne la définition de la variable aléatoire qui sera adoptée pour l'étude statistique et l'amélioration de la précision.

\section{Méthodologie}

De ce qui précède, on déduit qu'il faut utiliser la définition des pluies $n^{\circ} 1$, c'est-à-dire qu'il faut rechercher pour chaque épisode pluvieux enregistré, la pluie maximum tombée en 15 minutes, 30 minutes, 1 heure, etc. Comme on se place dans l'optique d'une étude complète, il ne faut pas se limiter à une durée mais tout calculer, depuis les durées les plus courtes compatibles avec le déroulement du pluviographe $(1 \mathrm{~h}$ pour un pluviographe à déroulement hebdomadaire) jusqu'à un ou plusieurs jours.

Dans la suite de l'étude on peut être amené à étudier la forme des pluies; on a donc intérêt à noter les temps de démarrage des pluies maxima pour pouvoir retrouver ensuite éventuellement le décalage entre la pluie de $1 \mathrm{~h}$ et celle de $2 \mathrm{~h}$, etc. et l'étudier pour déterminer la pluie de projet.

Insistons maintenant sur la grande variabilité des enregistrements de pluie : deux pluviomètres très proches peuvent donner des résultats différents de 20 à $25 \%$ sans qu'on voit bien la raison. Des courbes intensité-duréefréquence obtenues à partir d'un seul poste ne seront 
donc pas forcément représentatives et doivent être confirmées par d'autres observations.

Il est donc important de faire une étude systématique de tous les pluviographes de la région concernée, et donc de les recenser tous auparavant car très nombreux sont les organismes qui en installent, parfois pour une courte durée, mais parfois également pour une assez longue durée. Dans ces conditions, la critique des données recueillies devient une partie non négligeable de l'étude entreprise. Il n'existe d'ailleurs pas de méthode mathématique de critique des données (comme les doubles cumuls pour les pluies annuelles) et il faut se contenter d'analyser les enregistrements et de les comparer entre eux pour relever toutes les anomalies et les résoudre.

- Une fois qu'on a effectué le dépouillement de tous les pluviographes considérés comme valables (ce qui n'est pas déjà un petit travail) on essaye de les étudier statistiquement ; un problème se pose alors qui est celui du choix de la variable aléatoire qu'on va utiliser : il existe en effet trois définitions possibles :

- valeurs maxima mensuelles, saisonnières, ou annuelle,

- $N$ plus grandes valeurs en $N$ mois (ou $N$ saisons ou $N$ années) ;

- toutes les valeurs indépendantes supérieures à un seuil. On a donc $K$ valeurs pour $N$ mois (ou $N$ saisons ou $N$ années) avec $K \neq N$.

On se limitera ici aux deux premières définitions. En règle générale elles sont équivalentes à partir d'une durée de retour variable entre 2 ans et 10 ans. En dessous de cette durée de retour, la $1^{\text {ère }}$ définition conduit à une sous-estimation de la pluie puisqu'elle néglige des valeurs qui peuvent être importantes mais qui sont néanmoins inférieures au maximum mensuel (ou saisonnier ou annuel). Il faut donc alors adopter la $2^{\mathrm{e}}$ définition bien qu'elle soit d'un emploi plus malaisé.

- On peut maintenant constituer les échantillons de la variable aléatoire puisque le dépouillement effectué nous donne les maxima et les plus grandes valeurs. Il faut par contre décider si on travaille au niveau mensuel, saisonnier ou annuel. Utiliser le niveau annuel est plus simple, moins fatigant. Par contre on occulte ainsi les différences entre saisons (primordiales en climat méditerranéen) et on n'utilise pas à fond tous les renseignements foumis par le dépouillement. Si les séries sont vraiment de longue durée (supérieures à 20 ans) le niveau saisonnier doit être suffisant ; en dessous, on a intérêt à travailler par mois et à opérer des agglomérations par saisons.

La méthode est la suivante:

- On constitue les échantillons mensuels de la variable aléatoire choisie et pour toutes les durées envisagées dans le dépouillement.

- Pour chaque durée, on trace dans un graphique de probabilité (le graphique de Gumbel est le plus utilisé) les échantillons ordonnés en fonction de leur probabilité empirique $i / N+1$ ( $i$ rang de l'observation, $N$ taille de l'échantillon).

- On constitue des "saisons" à l'intérieur desquelles les lois mensuelles sont identiques. Cette définition des saisons est donc purement statistique bien qu'elle traduise forcément des saisons climatiques. On remarque d'ailleurs que pour des durées de pluie différentes les saisons ainsi définies ne sont pas forcément les mêmes.

- Comme les pluies sont indépendantes entre elles, on peut agglomérer les échantillons mensuels faisant partie de la même saison. On obtient un échantillon mensuel plus précis: pour une saison de 3 mois, à partir de 5 ans d'enregistrements, on détermine ainsi la loi mensuelle à partir d'un échantillon de 15 points. L'obtention de la loi théorique est plus fiable, mais il ne faut pas oublier que cette loi n'a pas été obtenue à partir de 15 ans d'enregistrements; il n'y a pas eu d'extension de l'information, mais une meilleure utilisation de l'information contenue dans les 5 ans de données.

- Des lois mensuelles obtenues à partir des échantillons mensuels agglomérés, on tire les lois saisonnières puis la loi annuelle; deux cas sont à envisager :

$I^{e r}$ cas : la variable aléatoire est le maximum mensuel ; la probabilité que le maximum saisonnier soit inférieur à $P_{0}$ est le produit des probabilités que chaque maximum mensuel soit inférieur à $P_{0}$. On a donc, si $F$ est la probabilité de non-dépassement:

$$
F_{\text {saison }}\left(P_{0}\right)=\pi_{i} F_{\text {mois } i}\left(P_{0}\right)
$$

Comme les saisons sont ici définies par l'identité des lois mensuelles, on a :

$$
F_{\text {saison }}\left(P_{0}\right)=\left[F_{\text {mois } i}\left(P_{0}\right)\right]^{k}
$$

où $k$ est le nombre de mois de la saison.

Ceci se traduit dans un graphique de Gumbel par une translation de Log $k$ parallèlement à l'axe des proba. bilités.

On a ensuite :

$$
F_{\text {année }}\left(P_{0}\right)=\pi_{i} F_{\text {saison } i}\left(P_{0}\right)
$$

$2^{e}$ cas : La variable aléatoire est donnée par l'échantillon des $N$ plus grandes valeurs. Plusieurs valeurs de cet échantillon ont pu se produire le même mois. On va donc utiliser la probabilité $G_{\text {mois } i}\left(P_{0}\right)$ que $P_{0}$ soit dépassée au moins $l$ fois au cours du mois $i$ (et non 1 fois et une seule). On a :

Prob $_{\text {saison }}\left(P>P_{0}\right.$ au moins 1 fois $)=$

Prob $\left[P>P_{0}\right.$ au moins 1 fois le mois 1

$$
\text { et } P \gtrless P_{0} \text { les autres mois] }
$$

+ Prob $\left[P<P_{0}\right.$ le mois 1 et $P>P_{0}$ au moins 1 fois le mois 2 et $P \lessgtr P_{0}$ les autres mois]

+ Prob $\left[P<P_{0}\right.$ les mois 1 et 2 , et $P>P_{0}$ au moins 1 fois le mois 3 et $P \lessgtr P_{0}$ les autres mois] $+\ldots$

D'où la formule :

$G_{\text {saison }}\left(P_{0}\right)=\sum_{i=1}^{k}\left(G_{\text {mois } i}\left(P_{0}\right) \prod_{j=1}^{i-1}\left[1-G_{\text {mois } j}\left(P_{0}\right)\right]\right)$

Vers les grandes valeurs de $P_{0}$, comme $G_{\text {mois }}\left(P_{0}\right)$ devient très petit, $G_{\text {saison }}\left(P_{0}\right)$ tend vers $k G_{\text {mois }}\left(P_{0}\right)$, on obtient donc très facilement une asymptote de la loi saisonnière. 


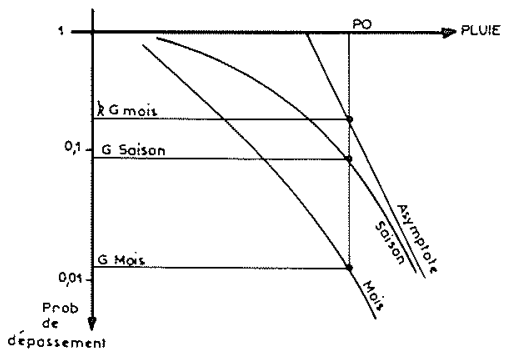

La loi annuelle, est donnée par la formule :

$$
\sum_{i}\left[G_{\text {saison } i}^{i-1}\left(1-G_{j=1}{ }_{\text {saison } j}\right)\right]
$$

et l'asymptote s'obtient là aussi très facilement par $\sum_{i} G_{\text {saison } i}$.

- L'analyse statistique précédente peut être faite pour chacune des stations dont on possède les enregistrements ; il est toutefois préférable d'adopter un point de vue synthétique sur l'ensemble des stations (par exemple pour la détermination des saisons) plutôt que de traiter séparément chaque station. On étudie de même la variation spatiale des lois statistiques obtenues en se souvenant que la loi obtenue à partir d'une station a une forte distribution d'échantillonnage, et que c'est surtout en regardant toutes les lois à la fois qu'on se fait une idée de la validité des résultats obtenus. Si cette confrontation indique qu'il existe un gradient, il faut alors le quantifier en étudiant les lois de chaque station sur la même période. Cette période commune à toutes les stations étant en général courte, on peut avoir intérêt à chiffrer ce gradient pluviométrique sur les pluies journalières (pluviomètre) pour lesquelles on possède en général une assez longue période commune.

\section{Etude partielle}

Nombreux sont les gens qui, pour les besoins d'un projet, réalisent une étude pluviométrique ponctuelle. Les résultats sont ensuite repris par d'autres et se propagent d'hydrologue en hydrologue suivant l'entregent de chacun. Ces résultats ne doivent pas à notre sens ètre utilisés sans critique pour les raisons suivantes :

- on ignore en général quelle définition des pluies a été utilisée ;

- on ignore souvent comment l'analyse statistique a été faite (au niveau mensuel, saisonnier ou annuel ?);

- on ne possède souvent que les résultats sous forme analytique, parfois sans fourchette de validité, et on ne peut donc pas savoir si l'adéquation de la loi théorique est bonne ou non.

- Le résultat provient de l'analyse d'une seule station, sans confrontation régionale, il est donc forcément approximatif à cause de la variabilité des mesures pluviométriques.

Le meilleur moyen de critiquer ces résultats est bien sûr de les reprendre en effectuant une étude complète comme indiqué ci-dessus. Mais cela n'est pas toujours possible (problème de temps, le but recherché ne néces- site pas forcément tout ce travail, etc.) ; aussi on va essayer de voir comment on peut, seulement à partir de pluviomètres, critiquer, étendre et utiliser des résultats tirés d'une étude pluviométrique déjà effectuée.

On suppose par exemple qu'on possède en $A$ les courbes intensité, durée, fréquence entre $1 \mathrm{~h}$ et $6 \mathrm{~h}$. On veut les utiliser en $B$ après critique et extension jusqu'à $24 \mathrm{~h}$. On veut également situer la période sur laquelle le dépouillement a été fait, savoir si elle est très pluvieuse ou très sèche. Si on ne possède pas de pluviomètre d'assez longue durée en $A$ et $B$, on doit faire une étude complète des pluies journalières de toute la région. $\mathrm{Si}$ on possède des renseignements pluviométrique en $A$ et $B$, on commence par étudier sur la période du dépouillement du pluviographe, les pluies journalières en $A$ (parfois aussi les pluies de $12 \mathrm{~h}$ si $A$ est une station climatologique). On rectifie la différence de définition des pluies en augmentant les résultats obtenus de 12 à $14 \%$ et on les compare sur un même graphique aux courbes intensité, durée, fréquence. On voit par exemple que pour Alençon (graphique 1) on a étendu les courbes $I D F$ jusqu'à $24 \mathrm{~h}$, alors que pour Grenoble le très mauvais raccordement indique qu'il vaut mieux ne pas utiliser ces courbes.

Il faut ensuite passer de $A$ en $B$, donc quantifier le passage des courbes $I D F$ de $A$ aux courbes $I D F$ de $B$. Pour cela on étudie les pluies journalières de $A$ et de $B$ sur leur plus longue période commune, et on en déduit les rapports de passage pour chaque fréquence. On est ensuite obligé d'admettre que ces rapports de passage sont valables pour d'autres durées.

On veut enfin savoir si la période sur laquelle on a calculé les courbes $I D F$ en $A$ est représentative ou non. Pour cela on cherche un critère caractérisant la pluviosité sur des durées de $24 \mathrm{~h}$ : le maxima annuel ne suffit pas bien sûr, mais on peut prendre par exemple la moyenne des 6 plus forts maxima mensuels, ou des 6 plus grandes pluies de chaque année. On trace ensuite la variation de cette grandeur année par année.

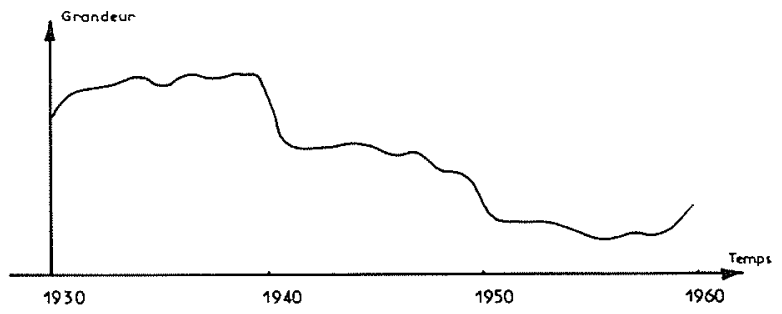

On en déduit si la période d'enregistrement des pluies était sèche (1950-1960) ou humide (1930-1940) ou moyenne (1940-1950).

\section{Exemple de la région Marseille-Avignon}

\section{Etude complète}

Pour le compte de la Ville d'Arles, nous avons effectué une étude ${ }^{(2)}$ pluviométrique complète de tous les

(2) Etudes hydrologique et hydraulique de l'assainissement pluvial des terres basses d'Arles. C.T.G.R.E.F. Division Irrigation, Ville d'Arles, Juin 1975. 

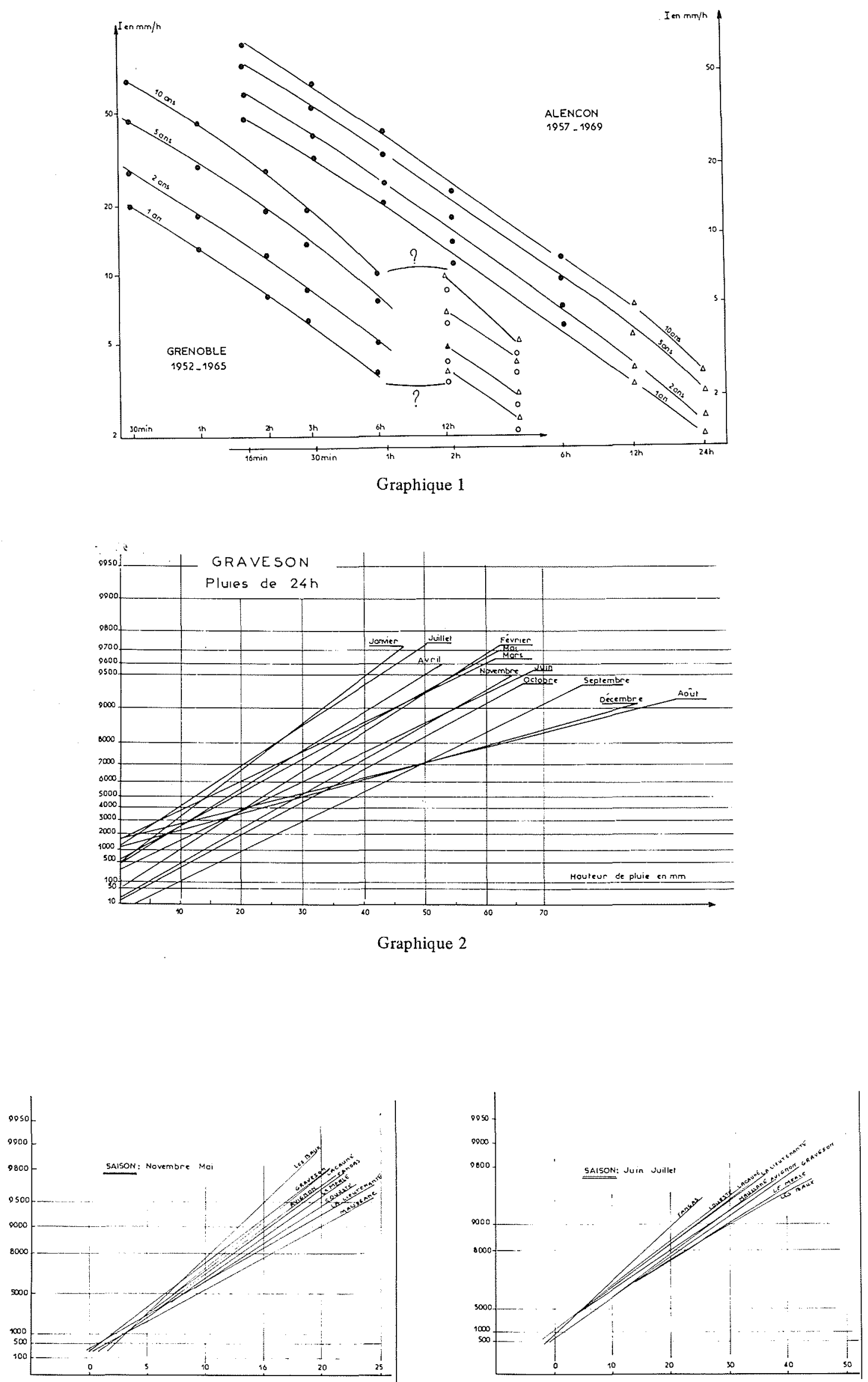

Graphique 3. - Variation spatiale des lois de hauteur de pluic. Durée : $1 \mathrm{~h}$. 
pluviographes du triangle Arles-Salon de Provence-Avignon. La liste des stations est la suivante :

\begin{tabular}{|c|c|c|c|c|}
\hline Numéro & Station & Organisme & $\begin{array}{c}\text { Période } \\
\text { d'observation }\end{array}$ & $\begin{array}{l}\text { Période } \\
\text { utilisée }\end{array}$ \\
\hline 1 & Arles & DDE 13 & $12 / 71-\left(^{*}\right)$ & - \\
\hline 2 & St Rémy & MN-DDE 13 & $01 / 65 \cdot\left({ }^{*}\right)$ & - \\
\hline 3 & Port St Louis & PC & $01 / 65-\left({ }^{*}\right)$ & - \\
\hline 4 & Chateaurenard & MN-PC & $01 / 65 \cdot\left({ }^{*}\right)$ & - \\
\hline 5 & Istres le Tubé & MN & (?) - & - \\
\hline 6 & Plan d'Orgon & EDF & $08 / 57-04 / 72$ & \\
\hline 7 & Graveson & EDF & $08 / 57-04 / 72$ & $08 / 57-04 / 72$ \\
\hline 8 & Avignon & EDF & $01 / 58-04 / 72$ & $01 / 58-04 / 72$ \\
\hline 9 & La Lieutenante & SRAE & $12 / 68-\left(^{*}\right)$ & $12 / 68-12 / 72$ \\
\hline 10 & Maussane & SRAE & $08 / 69-\left({ }^{*}\right)$ & $08 / 69 \cdot 12 / 72$ \\
\hline 11 & Courste & SRAE & $07 / 67-\left(^{*}\right)$ & $07 / 67-12 / 72$ \\
\hline 12 & Le Merle & SRAE & $11 / 68-\left(^{*}\right)$ & $11 / 68-12 / 72$ \\
\hline 13 & Fangas & SRAE & $10 / 67 \cdot\left({ }^{*}\right)$ & $10 / 67-12 / 72$ \\
\hline 14 & Les Baux & SRAE & $09 / 67-\left(^{*}\right)$ & $09 / 67-12 / 72$ \\
\hline 15 & La Caume & SRAE & $07 / 67-\left({ }^{*}\right)$ & $06 / 67-12 / 72$ \\
\hline
\end{tabular}

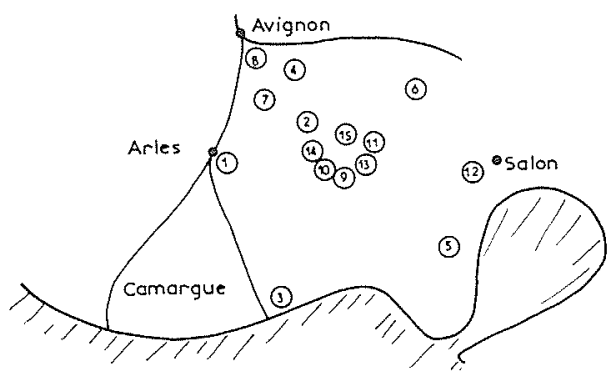

De cette information abondante nous n'avons pu utiliser que 9 postes ( $n^{\text {os }} 7$ à 15 ) qui sont d'assez courte durée, sauf Graveson et Avignon (14 à 15 ans). Nous avons commencé par voir à partir de quelle durée de retour les deux définitions possibles de la variable aléatoire étaient équivalentes : une comparaison graphique a montré que c'est entre 3 et 7 ans que les échantillons deviennent confondus. Comme nous nous intéressions à des durées de retour supérieures à 10 ans, nous avons utilisé les maxima mensuels. Après vérification que la loi de Gumbel s'adaptait bien à la majorité des échantillons mensuels, nous l'avons utilisée pour la séparation en saisons. Le graphique 2 montre qu'il n'est pas toujours facile de choisir ce découpage en saison. Le mois de juillet pour les pluies de $24 \mathrm{~h}$ est par exemple difficile à situer. Il faut en effet tenir compte de l'échantillonnage mais également ne pas regrouper des mois trop dissemblables. Après comparaison des graphiques de toutes les durées, pour toutes les stations, nous avons adopté les saisons suivantes :

\begin{tabular}{|c|l|}
\hline Durées & \multicolumn{1}{|c|}{ Saisons } \\
\hline $1.2 .3 \mathrm{~h}$ & Nov-Mai, Juin-Juil, Août-Octobre \\
$6 \mathrm{~h}$ & Nov-Juil, Août-Octobre \\
$12 \mathrm{~h}$ & Nov-Déc, Janv-Juil, Août-Octobre \\
$24 \mathrm{~h}$ & Janv-Juil, Août-Décembre \\
\hline
\end{tabular}

Il est alors possible de comparer par saisons la dispersion spatiale (graphique 3) entre toutes les stations. On ne repère pas de gradient systématique : la station Les Baux qui a les pluies de $1 \mathrm{~h}$ les plus faibles en Novembre-Mai, a par contre les pluies les plus fortes en juin-juillet. On peut donc adopter les résultats des stations longue durée, c'est-à-dire Avignon ou Graveson. Par le passage des lois mensuelles aux lois saisonnières puis annuelles, on obtient les courbes finales du graphique 4 . On peut se poser la question des limites de validité de ces courbes : on peut aller un peu au-delà de 20 ans vers les grandes durées de retour puisque la taille de l'échantillon est de 15 ans. Vu les faibles durées de retour, on a dit ci-dessus qu'on ne devrait pas aller en dessous de 7 ou 8 ans puisqu'on travaille sur les maxima. Ceci est vrai au niveau mensuel, alors que la combinaison des lois mensuelles permet d'aller sur la loi annuelle à une durée de retour inférieure à 2 ans, ainsi qu'on peut le vérifier sur le graphique 4 pour les durées de $1 \mathrm{~h}$ et $24 \mathrm{~h}$. On obtient donc en définitive le graphique 5 qui donne les mêmes résultats sous une présentation plus familière.

Pour apprécier la période sur laquelle on a effectué le dépouillement du pluviographe, on a tracé sur le graphique 6 la variation de la moyenne des maxima mensuels des pluies journalières pour toutes les années pour lesquelles on possède plus de 9 mois valables. On voit que la période 1958-1972 n'est pas beaucoup plus pluvieuse en moyenne que la période 1935-1972. Les courbes obtenues peuvent être considérées comme représentatives.

\section{Etude partielle}

La région d'Aix-Marseille est en plein développement à l'heure actuelle et nombreux sont les bureaux d'étude qui y réalisent des dépouillements de pluviographes pour dimensionner les réseaux d'assainissement de 
Graphique 4

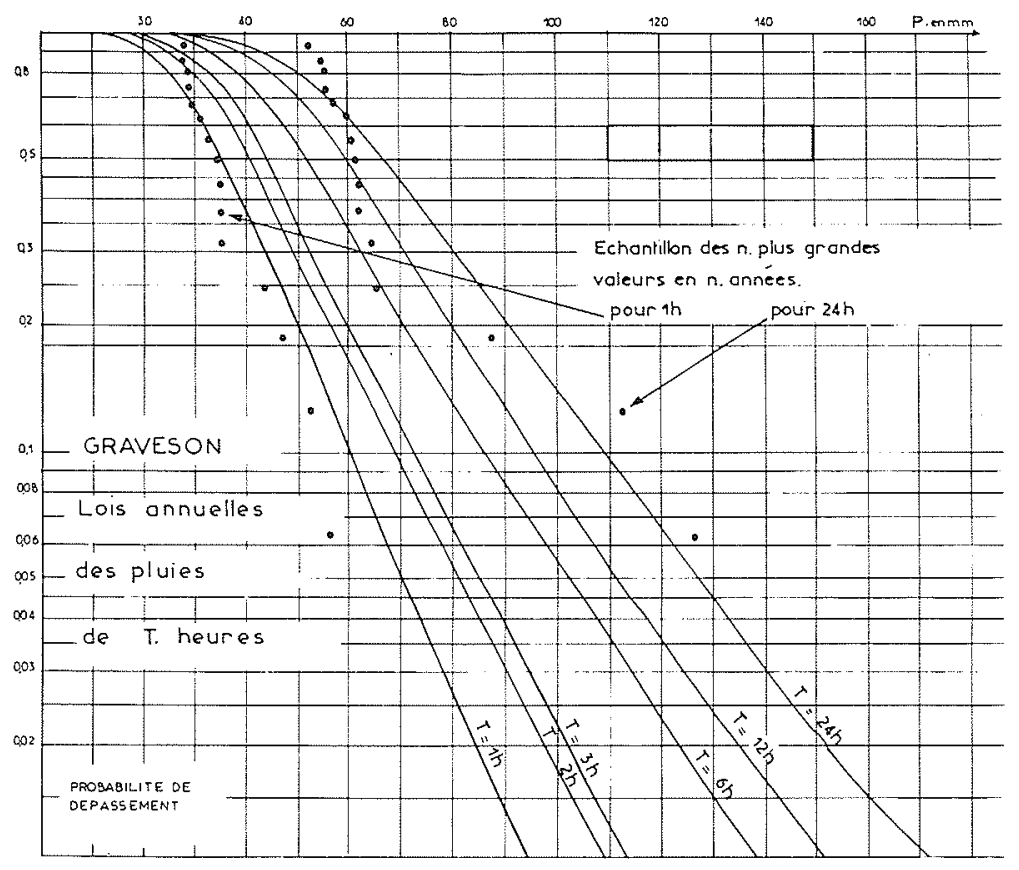

Graphique 5

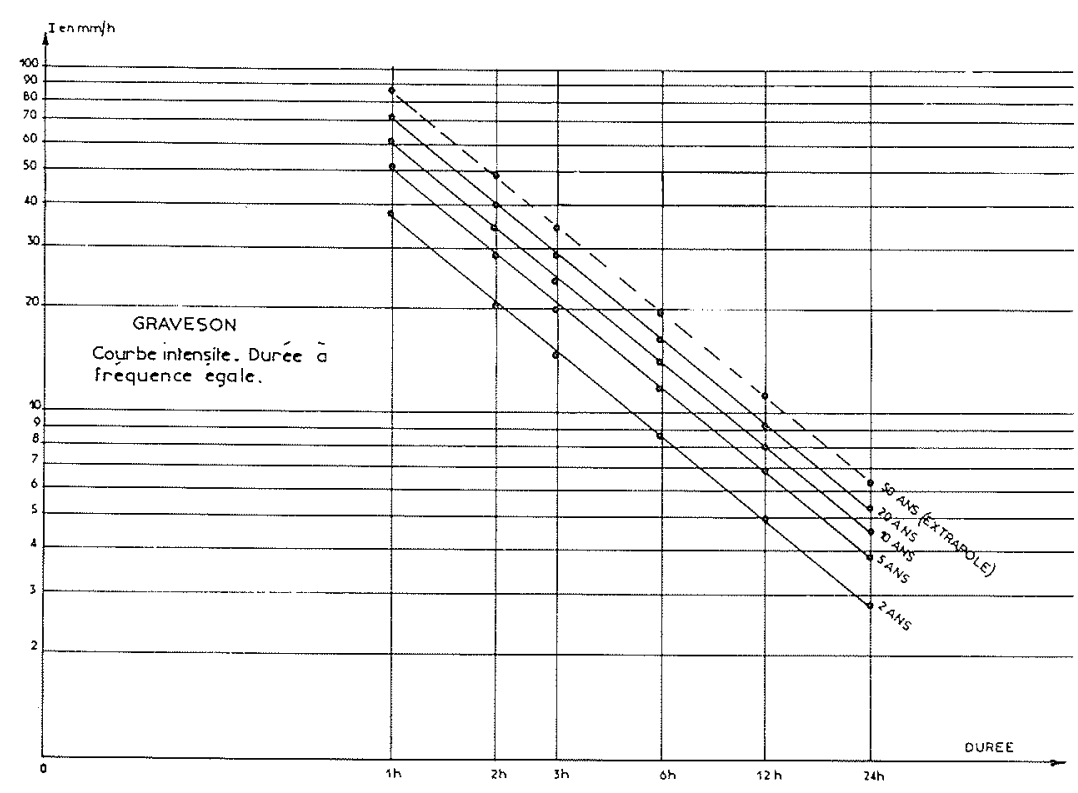

-

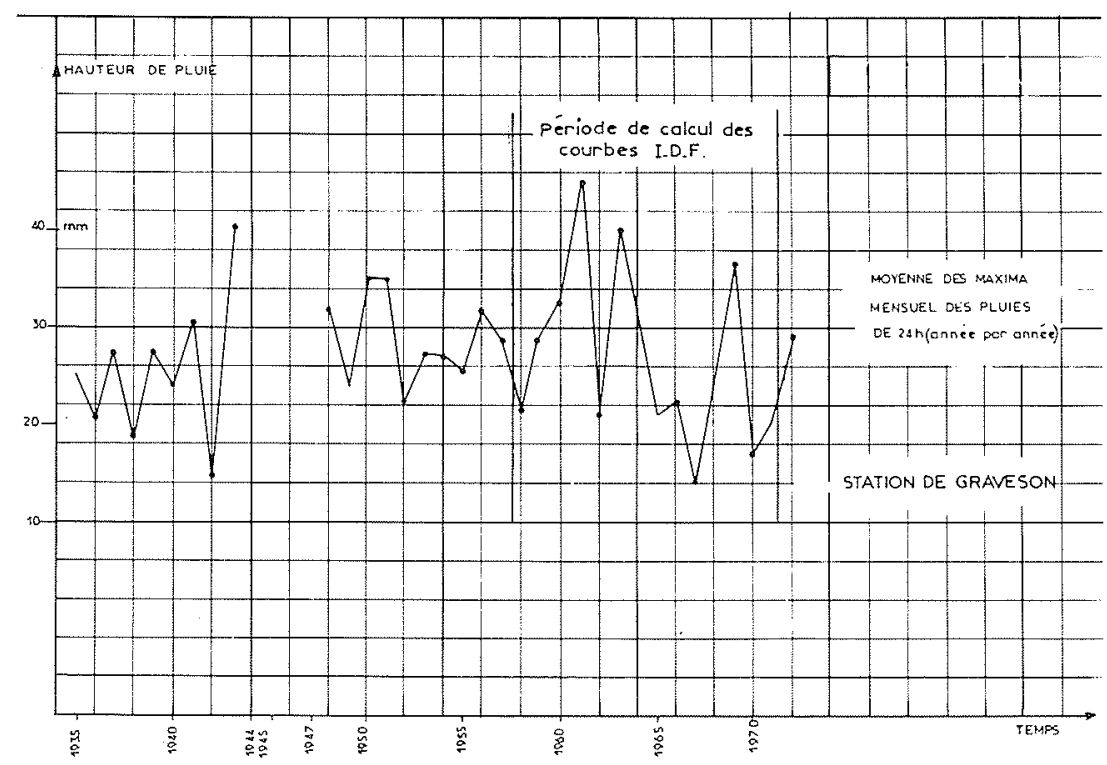




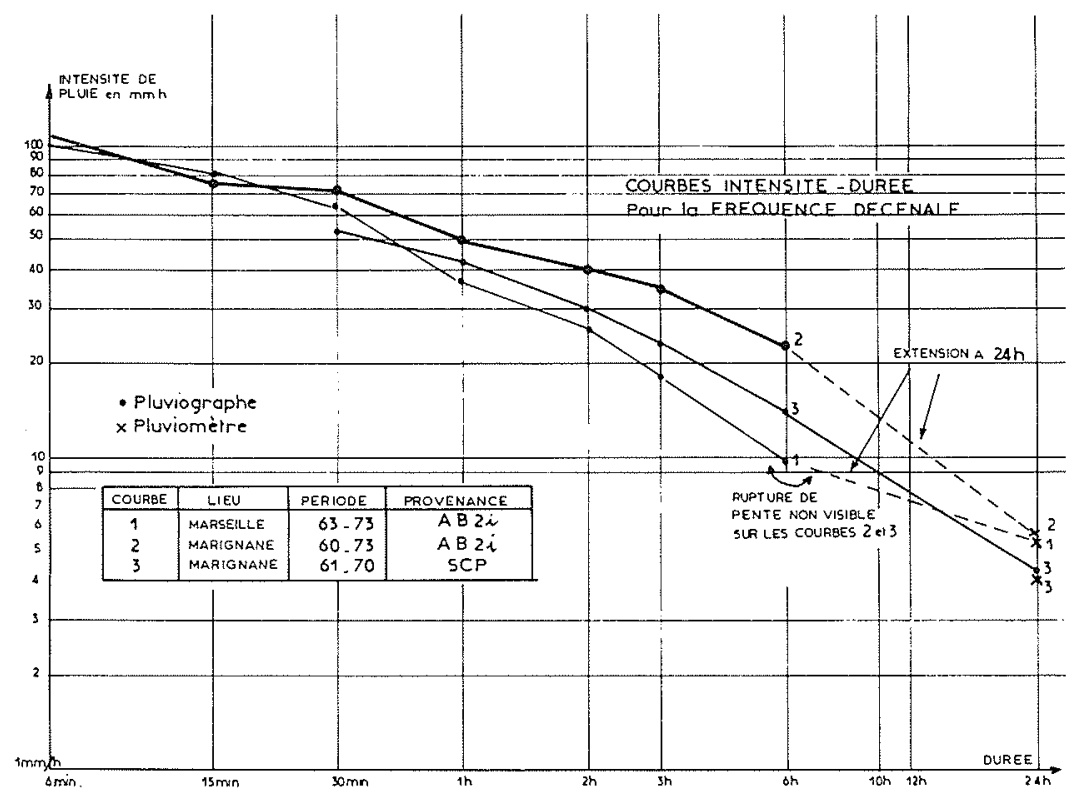

Graphique 7

Courbes intensité - Durée. Pour la fréquence décénale

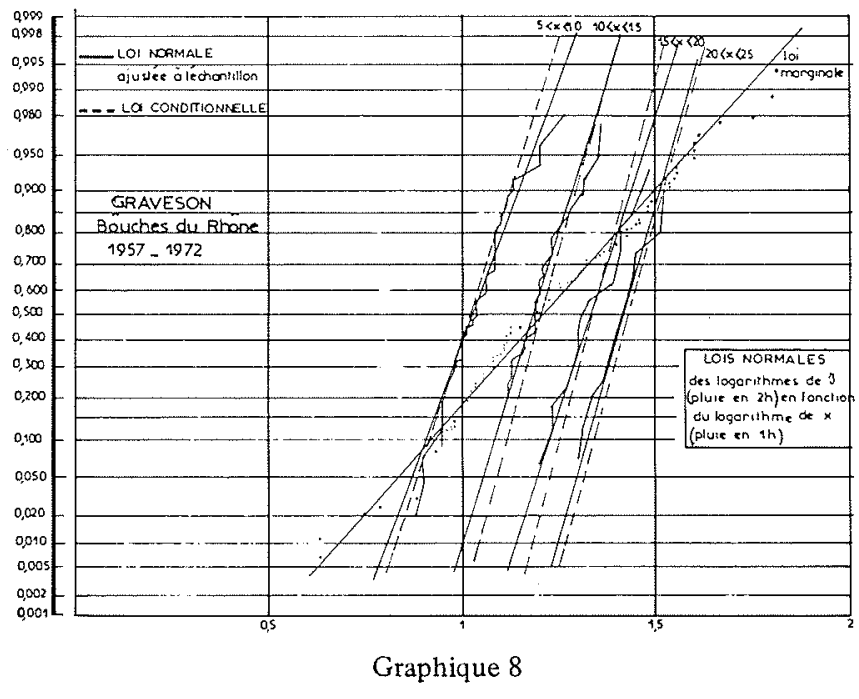

Lois normales des logarithmes de $\mathfrak{I}$ (pluie en $2 \mathrm{~h}$ ) en fonction du logarithme de $\times$ (pluie en $1 \mathrm{~h}$ )

\section{Graphique 9}

Rapport des hauteurs de pluie $\mathrm{Y} / \mathrm{X}$ en fonction de la hauteur $\mathrm{X}$ et des durées de $X$ et $Y$ région méditerranéenne.

$\mathrm{Y}$ est la pluie médiane encadrant la pluie $\mathrm{X}$ ou intérieure à $\mathrm{X}$ selon qu'elle est plus grande ou plus petite que $X$. Les différents graphiques correspondent à différentes durées de $X$. Les courbes sont paramétrées par la durée de Y.
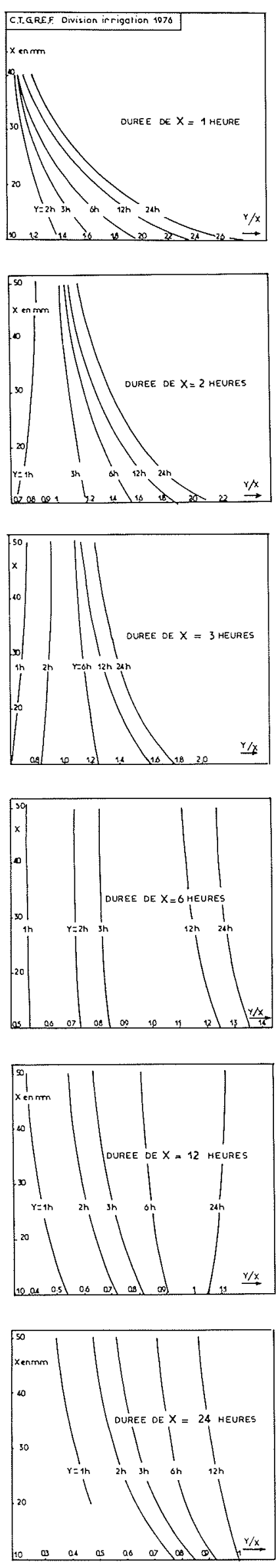
telle $Z U P$ ou $Z A D$ ou $Z I$ ou $Z A C$. Malheureusement aucune étude synthétique n'a été faite au niveau régional, et on est conduit à utiliser les courbes $I D F$ que l'on trouve dans la littérature ou celles que Godard a faites pour Montpellier. Essayons de voir, et le plus simplement possible, ce qu'on peut faire avec de telles données élaborées : nous possédons la courbe de la pluie décennale à Marignane (période 1961-1970) provenant de la Société du Canal de Provence et d'Aménagement de la Région Provençale, et les courbes de l'intensité décennale à Marseille (1963-1973) et à Marignane (1960-1973) provenant du Bureau d'études $\mathrm{AB} 2 \mathrm{i}$.

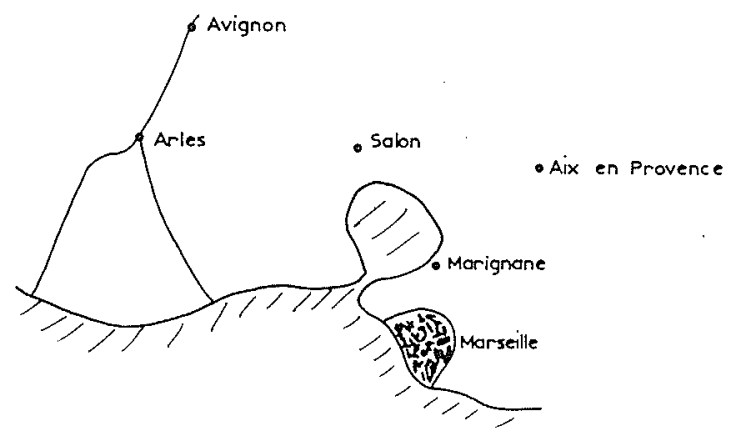

Rapportées dans le même graphique 7 , ces 3 courbes font apparaître des différences sensibles (de 1 à 2 entre Marseille et Marignane pour des durées de $6 \mathrm{~h}$ et pour des périodes de dépouillement voisines).

Pour savoir si une telle différence est plausible, on a comparé les lois des maxima journaliers annuels, d'une part sur la plus grande période commune (49-65), et d'autre part sur les périodes particulières du dépouillement des pluviographes. Ces comparaisons ont montré une faible variation spatiale, et une grande variabilité des lois statistiques en fonction de la période du dépouillement. Donc sur le graphique 7, si l'écart entre les courbes 1 et 2 est anormal, celui qui sépare les courbes 2 et 3 ne l'est pas. Le report dans ce graphique des pluies décennales de $24 \mathrm{~h}$ (après correction de 1,14 pour tenir compte de la définition des pluies obtenues à partir d'un pluviomètre) permet d'éliminer la courbe 1 pour la rupture de pente qu'elle présente aux alentours de la pluie de $6 \mathrm{~h}$.

En conclusion, on n'a pas intérêt à utiliser pour Marseille la courbe 1, mais une des courbes de Marignane. Il faudrait encore pour en choisir une situer les deux périodes de dépouillement à l'intérieur d'une période plus grande. Signalons toutefois que la courbe 3 est très proche de la courbe correspondante obtenue à partir de Graveson et qu'on doit donc pouvoir la considérer comme valable pour toute la région qui s'étend d'Avignon à Marseille. Bien sûr ceci demanderait à être confirmé par une étude des pluies journalières de tous les postes de cette région.

\section{Structure fine de la pluie}

L'étude de la répartition de la pluie à l'intérieur d'un épisode pluvieux est utile pour déterminer la "pluie de projet", c'est-à-dire pour répartir la pluie moyenne sur une durée déterminée à l'intérieur de cette durée. Mais la connaissance de cette répartition doit également permettre de tenir compte de la pluie tombée en dehors de cette durée : si un bassin versant a un temps caractéristique de $6 \mathrm{~h}$, on se contente, en général, d'étudier les pluies de $6 \mathrm{~h}$ et on néglige ce qui se passe avant et après.

Ce problème a déjà été abordé par Normand (3) et par Desbordes, Vanky et Lavabre ${ }^{(4)}$. La méthode que nous proposons part du point de vue synthétique suivant : un épisode pluvieux est un phénomène aléatoire caractérisé par plusieurs variables, liées ou non. $\mathrm{Au}$ lieu d'étudier séparément chaque variable, on peut essayer de les étudier ensemble et avec leurs liaisons. La loi normale à plusieurs variables est bien sûr la loi la plus facile à utiliser. Une première étape de la méthode consiste donc à opérer des transformations sur les variables telles que la loi des $n$ variables transformées soit une loi normale à $n$ variables. Supposons que l'on connaisse les méthodes permettant de calculer les paramètres et les quantiles de cette loi normale à $n$ variables (c'est bien sûr une hypothèse de taille). On commence à trouver par tatonnements successifs les transformations qui donnent la loi normale la plus adaptée à l'échantillon. On utilise ensuite les propriétés de la loi normale pour quantifier la pluie de projet présentant les caractéristiques souhaitées.

Le problème se formule ainsi : si la pluie est caractérisée par les paramètres $x_{1}, x_{2}, \ldots x_{n}$, admettons qu'on ait trouvé la loi $F\left(x_{1}, x_{2}, \ldots x_{n}\right)$. Il est rare que tous les paramètres aient la même importance, et qu'on demande de quantifier la probabilité pour qu'on ait, en même temps, $x_{1}>X_{1}, x_{2}>X_{2}$, etc. On aurait d'ailleurs du mal à dégager clairement le sens de cette probabilité. En général un paramètre $x_{1}$ est privilégié. Pour la durée de retour choisie on fixe la valeur de $x_{1}$ (soit $X_{1}$ ) par l'étude de sa loi marginale et on étudie ensuite les autres paramètres par leur loi conditionnelle, liée par $x_{1}=X_{1}$. Assez souvent d'ailleurs on n'aura besoin que des quantiles 0,5 de cette loi conditionnelle puisque c'est par la variable $x_{1}$ qu'on quantifie le phénomène pour la durée de retour choisie. Cette remarque va nous être très utile dans ce qui suit : supposons par exemple qu'on veuille calculer le débit d'un bassin versant de temps caractéristique $6 \mathrm{~h}$ pour une durée de retour 20 ans. Le paramètre le plus important est la pluie de $6 \mathrm{~h}$. En général on se contente de calculer la valeur $X$ de la pluie de $6 \mathrm{~h}$ de durée de retour 20 ans. Supposons également qu'on veuille tenir compte de la pluie qui encadre cet intervalle de $6 \mathrm{~h}$; on essaie alors d'étudier la pluie de $12 \mathrm{~h}$. Mais les pluies de $6 \mathrm{~h}$ et celles de $12 \mathrm{~h}$ sont liées. Comme la durée de retour de 20 ans est prise en compte par la fixation de la pluie de $6 \mathrm{~h}$ à la valeur $X$, on n'a pas de raison de se placer du côté de la sécurité, ou non, pour fixer la pluie de $12 \mathrm{~h}$. On cherche donc la valeur $Y$ de la pluie de $12 \mathrm{~h}$ qui a $50 \%$ de chances d'être dépassée sachant que la

(3) Modèles pour l'étude du ruissellement urbain. Houille Blanche $3 / 71$.

(4) Réflexions sur les méthodes de calcul des réseaux d'assainissement urbain. $4^{\mathrm{e}}$ partie. Pluie de projet : Résultat des recherches. Juillet 1973 . 
pluie de $6 \mathrm{~h}$ du même épisode pluvieux est égale à $X$. Si les pluies de $6 \mathrm{~h}$ et celles de $12 \mathrm{~h}$ suivent, après transformations, une loi normale à deux variables $(x, y)$, la loi marginale de $x$ (pluies de $6 \mathrm{~h}$ ) fixera $X$, et la loi conditionnelle de $y$ pour $x$ fixé, donnera $Y$.

La corrélation entre pluies de $6 \mathrm{~h}$ et pluies de $12 \mathrm{~h}$ est en général bonne puisque la pluie de $12 \mathrm{~h}$ contient la pluie de $6 \mathrm{~h}$. De façon plus fine on peut donc essayer de relier la pluie de $6 \mathrm{~h}$ au supplément de pluie apporté dans les autres $6 \mathrm{~h}$ de l'intervalle de $12 \mathrm{~h}$. Le coefficient de corrélation peut alors être nul, ce qui ne change rien à ce qui est dit ci-dessus, puisque la loi normale $F(x, y)$ dégénère en un produit de 2 lois normales à 1 variable. La loi conditionnelle de y est la loi marginale.

L'étude du phénomène à deux variables n'est quand même pas suffisante : en reprenant l'exemple ci-dessus, une fois qu'on connait $X$ et $Y$, il faudrait connaitre le décalage qui sépare le début de la pluie de $6 \mathrm{~h}$ du début de la pluie de $12 \mathrm{~h}$ pour obtenir une chronique de pluie. Si $t$ est ce décalage, on est conduit à étudier la loi $F(x, y, t)$ des trois variables liées. Supposons que ces trois variables suivent, après transformations, une loi normale à 3 variables. La loi marginale de $x$ permet de fixer $X$ pour la durée de retour 20 ans. De même que ci-dessus on va fixer $y$ et $t$ pour la probabilité 0,5 . Mais $y$ et $t$ suivent maintenant une loi normale à deux variables qui est une loi conditionnelle de $F(x, y, t)$ liée par $x=X$. Soit $F(y, t / x=X)$ cette loi normale conditionnelle. Elle possède deux lois marginales à 1 variable $G(y / x=X)$ et $G(t / x=X)$. Repartons maintenant de la loi générale $F(x, y, t)$. Elle possède 3 lois marginales à 2 variables. Prenons celle qui relie $x$ et $y$ soit $G(x, y)$. Si on fixe $x$ à $X$, elle nous donne la loi conditionnelle de $y$ liée par $x=X$. La commutativité des deux opérateurs "conditionnel" et "marginal" nous permet d'affirmer que cette loi de $y$ n'est autre que la loi $G(y / x=X)$.

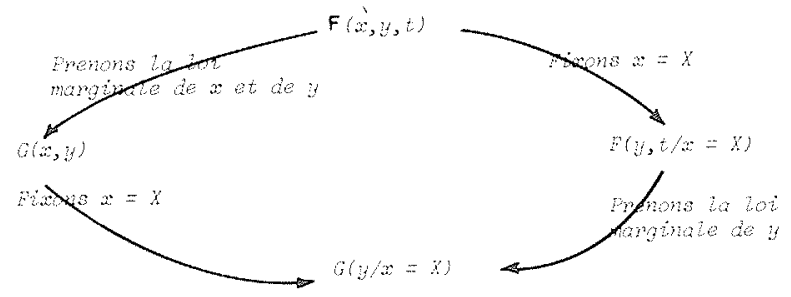

Cette première propriété montre comment, même avec trois variables, on peut se ramener à l'étude des lois à deux variables. Reprenons notre exemple : nous supposons toujours que $x, y$ et $t$ suivent une loi normale à 3 variables, mais nous n'étudions que les deux lois marginales $G(x, y)$ et $G(x, t)$, d'où nous déduisons les deux lois conditionnelles $G(y / x=X)$ et $G(t / x=X)$. Soient $Y$ et $T$ les quantiles 0,5 de ces deux lois marginales. Si nous reprenons la loi conditionnelle à deux variables $F(y, t / x=X)$ et si nous en prenons la loi conditionnelle de $t, F(t / x=X, y=Y)$, le fait que toutes ces lois soient des lois normales permet d'affirmer que le quantile 0,5 de cette loi doublement conditionnelle est́t précisément $T$. On obtient donc le schéma ci-après.

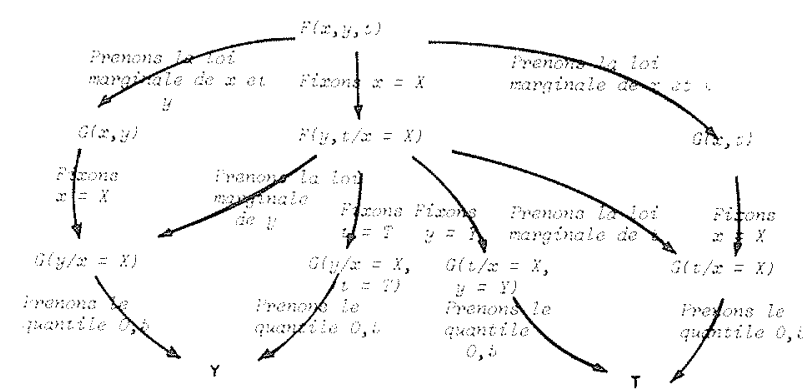

On voit qu'on peut déterminer $Y$ et $T$ sans tenir compte de la liaison qui existe entre $y$ et $t$. Mais cela n'est vrai que parce qu'on s'est limité à la détermination des quantiles 0,5 .

Si ce raisonnement peut être étendu aux lois normales à $n$ variables, la détermination de la pluie de projet peut se faire en quantifiant d'abord le paramètre principal pour la durée de retour choisie et les autres paramètres par le quantile 0,5 des lois normales conditionnelles déduites des lois normales à 2 variables que suivent le paramètre principal et chacun des autres paramètres.

On peut donc essayer de dresser des courbes donnant le quantile 0,5 des lois conditionnelles qui permettrait connaissant le paramètre principal d'en déduire les autres:

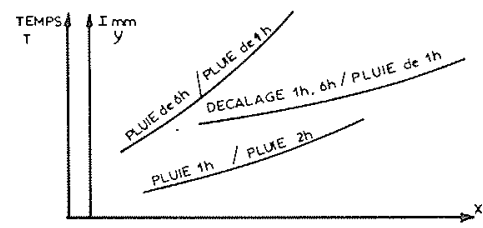

Ces courbes ne traduisent en fait que la droite de régression reliant les variables transformées entre elles. Elles sont des droites horizontales lorsque la corrélation entre variables est nulle. Ce qui est toutefois primordial et limite pour l'instant l'utilisation de cette méthode, c'est la vérification que les variables transformées suivent ensemble une loi normale.

Exemple : Nous avons essayé de calculer les courbes ci-dessus pour la station de Graveson. En fait nous n'avons pas pu les établir pour les temps de décalage entre débuts de pluie car ces données n'avaient pas été recueillies lors du dépouillement. Le graphique 8 montre que, pour les pluies de $1 \mathrm{~h}$, les logarithmes des pluies suivent bien les lois conditionnelles théoriques. Le coefficient de corrélation est égal à 0,95312 .

\begin{tabular}{|c|l|l|l|l|l|}
\hline \multicolumn{2}{|c|}{$\begin{array}{c}\text { Pluie de 1 } \mathrm{h} \\
\text { (valeurs naturelles) }\end{array}$} & 5,10 & 10,15 & 15,20 & 20,25 \\
\hline & $\begin{array}{l}\text { Moyenne } \\
\text { calculé } \\
\text { Moyenne }\end{array}$ & 1,03 & 1,18 & 1,33 & 1,41 \\
$\begin{array}{l}\text { Pluies } \\
\text { de 2 } \mathrm{h} \\
\text { théorique } \\
\text { (logarithmes) }\end{array}$ & $\begin{array}{l}\text { Ecart-type } \\
\text { calcuié } \\
\text { Ecart-type } \\
\text { théorique }\end{array}$ & 0,025 & 1,22 & 1,34 & 1,43 \\
\hline
\end{tabular}


Il est bien évident que nous n'avons pu faire ces vérifications que pour les valeurs centrales (nous avons pris les classes ayant plus de 10 données).

Le tableau des coefficients de corrélation est le suivant :

\begin{tabular}{|c|c|c|c|c|c|c|}
\hline 1 & 1 & 2 & 3 & 6 & 12 & 24 \\
\hline 2 & & 0,95 & 0,89 & 0,74 & 0,60 & 0,52 \\
\hline 3 & & & 0,97 & 0,86 & 0,73 & 0,66 \\
\hline 6 & & & & 0,93 & 0,81 & 0,75 \\
\hline 12 & & & & & 0,93 & 0,88 \\
\hline 24 & & & & & & \\
\hline
\end{tabular}

On voit que la corrélation devient de plus en plus médiocre quand les durées s'écartent l'une de l'autre.

L'ensemble des résultats étant malgré tout satisfaisant nous avons tracé le graphique 9 qui synthétise les résultats. Il serait d'ailleurs assez intéressant d'établir ces mêmes graphiques par saison et d'en apprécier les différences.

Signalons enfin que ces graphiques sont cohérents. Par exemple, si on se donne comme point de départ une pluie en $24 \mathrm{~h}$ de $26 \mathrm{~mm}$, on peut en déduire toutes les pluies de durée inférieure à partir du graphique 9 . Mais la pluie de $12 \mathrm{~h}$ ainsi déterminée permet également de fixer les pluies de durée inférieure. Comparons les résultats :

\begin{tabular}{|c|c|c|c|c|c|}
\hline $\begin{array}{ll}\text { déterminée } & \text { Pluie de } \\
\text { à partir de } & \end{array}$ & $12 \mathrm{~h}$ & $6 \mathrm{~h}$ & $3 \mathrm{~h}$ & $2 \mathrm{~h}$ & $1 \mathrm{~h}$ \\
\hline $\begin{array}{l}\text { Pluie de } 24 \mathrm{~h} \\
=26 \mathrm{~mm}\end{array}$ & 24 & 20,4 & 17,3 & 15 & 11,3 \\
\hline $\begin{array}{l}\text { Pluie de } 12 \mathrm{~h} \\
=24 \mathrm{~mm}\end{array}$ & & 20,5 & 17,3 & 15 & 11,2 \\
\hline $\begin{array}{l}\text { Pluie de } 6 \mathrm{~h} \\
=20,5 \mathrm{~mm}\end{array}$ & & & & 14,9 & 11,1 \\
\hline $\begin{array}{l}\text { Pluie de } 3 \mathrm{~h} \\
=17,2 \mathrm{~mm}\end{array}$ & & & & 15 & 10,9 \\
\hline $\begin{array}{l}\text { Pluie de } 2 \mathrm{~h} \\
=15 \mathrm{~mm}\end{array}$ & & & & & 11 \\
\hline
\end{tabular}

\section{Abattement des pluies}

Sous ce titre, on s'intéresse au coefficient qui permet de passer de la pluie ponctuelle d'une durée de retour donnée à la pluie moyenne sur une surface de même durée de retour. C'est ce coefficient qui est en général dénommé "Coefficient d'abattement".

\section{Théorie et critique}

Signalons tout de suite que dans notre optique, qui est de calculer des débits, il ne faut pas utiliser les coefficients qu'on trouve dans la littérature (Grisollet, Frulhing, Richards) qui sont calculés à partir de la pluie maximale au centre de l'orage. En effet, la loi statistique de la pluie ponctuelle $P$; soit $P_{M}=C(S, P) \times P$ où pluviométrique ou pluviographique quelconque nullement situé au centre de tous les orages. En utilisant ces coefficients on sous-estime donc forcément les résultats.

Par ailleurs, ces méthodes donnent en général un coefficient variable avec la superficie envisagée, mais indépendant de la pluie ponctuelle. Or on peut penser que l'importance de la quantité d'eau recueillie au pluviomètre dépend de sa distance au centre de l'orage. Le coefficient d'abattement doit donc décroitre quand la hauteur de pluie croit. Il faut donc envisager un coefficient d'abattement $C$ fonction de la surface $S$ et de la pluie ponctuelle $P$; soit $P_{M}=C(S, P) \times P$ où $P_{M}$ est la pluie moyenne sur la surface considérée, de même durée de retour que la pluie ponctuelle $P$.

Nous utilisons la méthode de Brunet-Moret et Roche ${ }^{(5)}$ que nous rappelons brièvement : une hypothèse de départ est que les lois ponctuelles de tous les postes pluviométriques sont les mêmes (hypothèse d'isotropie). On peut alors calculer la densité de probabilité empirique $\rho\left(P, P_{M}\right)$ du couple pluie moyennepluie ponctuelle en mélangeant les couples $P, P_{M}$ des différents postes pluviométriques. Les lois marginales de densités

$f(P)=\int_{0}^{\infty} \rho\left(P, P_{M}\right) d P_{M}$ et $g\left(P_{M}\right)=\int_{0}^{\infty} \rho\left(P, P_{M}\right) d P$

permettent en principe de calculer le coefficient d'abattement comme rapport des quantiles de ces deux lois. En fait, ces lois sont obtenues à partir du petit nombre d'années pour lesquelles on possède des informations sur tous les pluviomètres, et il arrive souvent qu'on puisse obtenir la loi $f(P)$ en un poste (appelé station longue durée) pour lequel on dispose d'un plus grand nombre de données soit $f_{1}^{\prime}(P)$. Les auteurs de la méthode proposent donc d'utiliser une densité modifiée $\rho \times \frac{f_{1}}{f}$ qui tient compte de la loi longue durée. On en déduit la densité de la nouvelle loi marginale de la pluie moyenne:

$$
g_{1}\left(P_{M}\right)=\int_{0}^{\infty} \rho\left(P, P_{M}\right) \times \frac{f_{1}(P)}{f(P)} d P
$$

et on calcule enfin le coefficient d'abattement à partir des lois de densité de probabilité $f_{1}$ et $g_{1}$.

Nous avons voulu appliquer cette méthode à partir des postes pluviométriques qui existent sur la région Sud d'Avignon et les résultats obtenus nous ont obligé à en cerner certaines limites. Certains résultats indiqués ci-après sont partiels car nous n'avons pas pour projet de critiquer cette méthode mais plutôt de calculer un coefficient d'abattement.

(5) Brunet-Moret, Roche : Etude théorique et méthodologique de l'abattement des pluies (cahiers de I'ORSTOM Série hydrologie n $n^{\circ}$ /1966) 

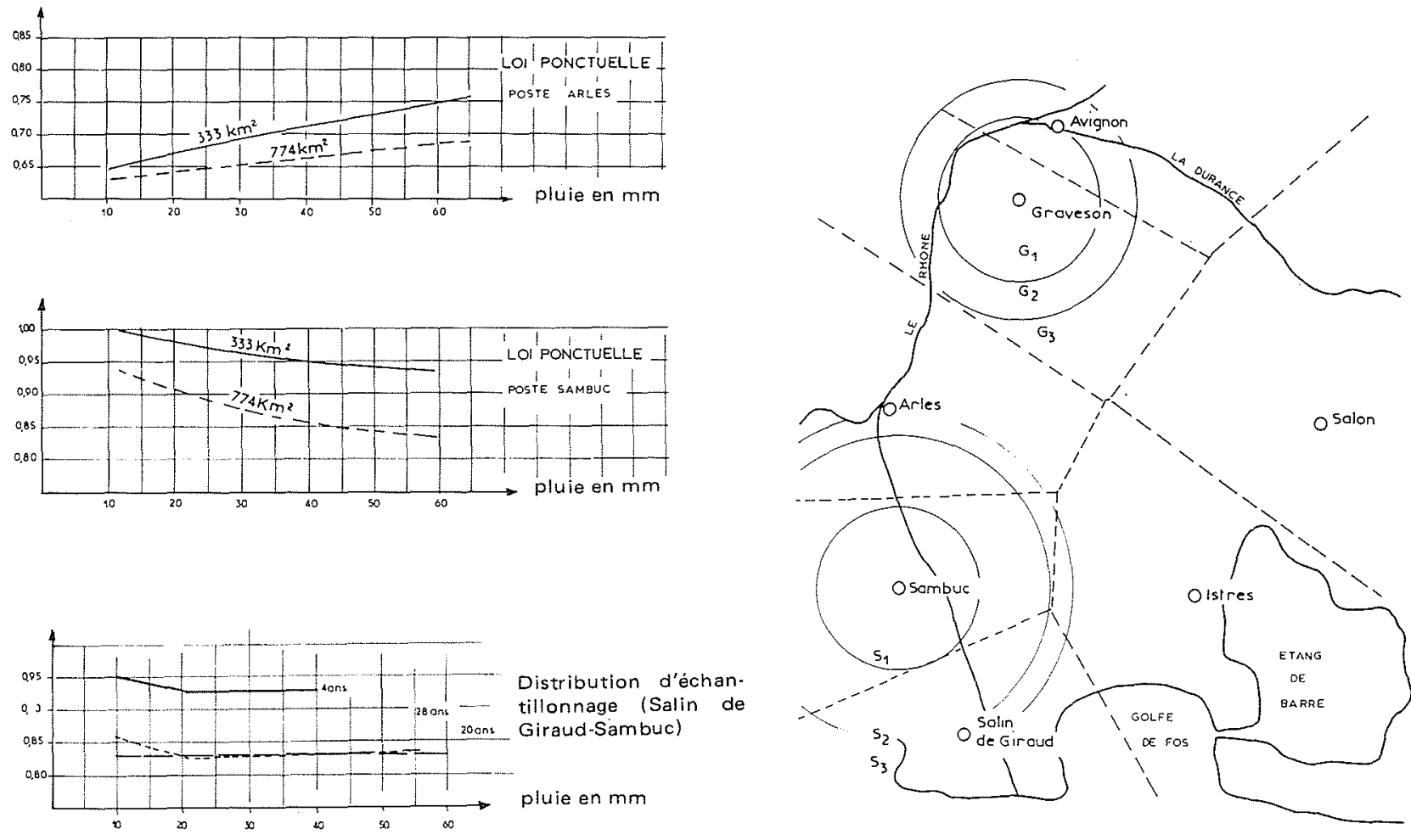

Graphique 10. - Coefficient d'abattement.

Graphique 11. - Schéma de situation. Postes pluviométriques et surfaces de Thiessan. (Attention les échelles ne sont pas respectées).
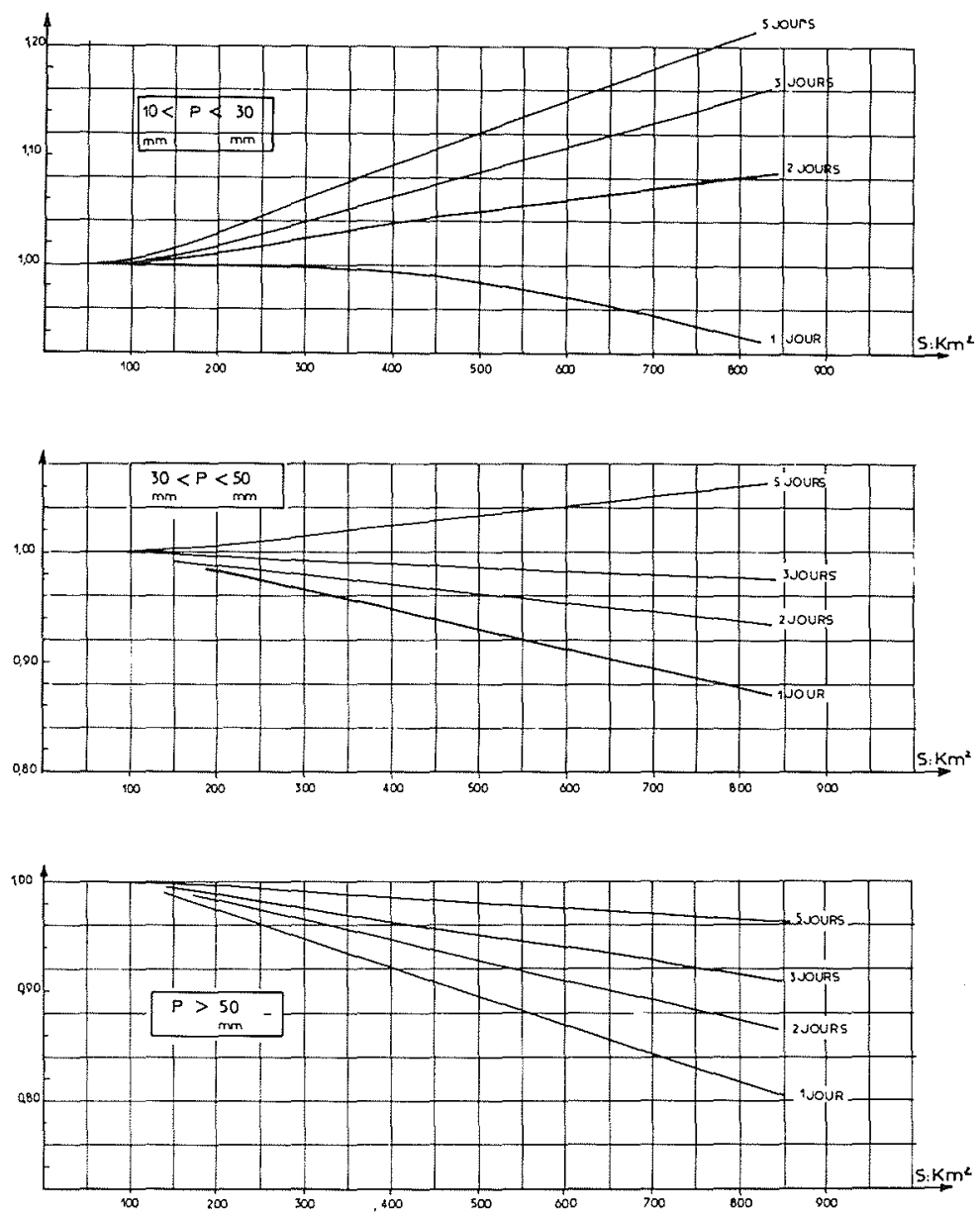

Graphique 12. - Coefficient d'abattement. Station de Graveson (Bouches du Rhône). 


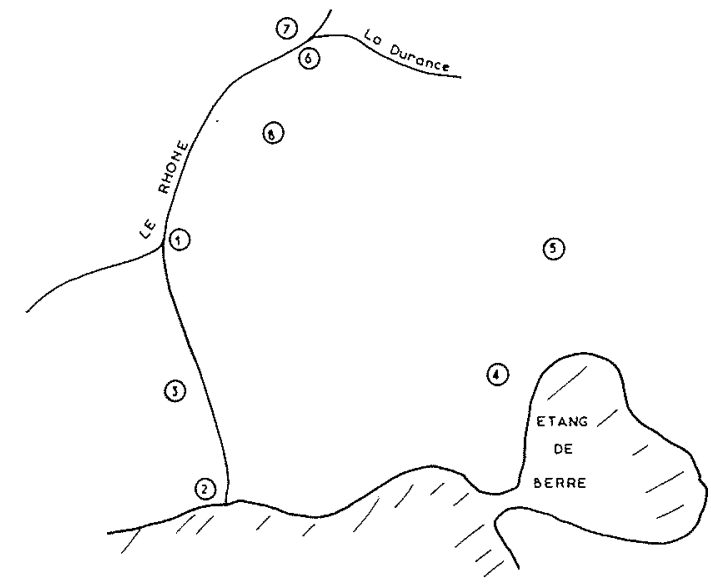

\begin{tabular}{|c|l|c|c|c|}
\hline$N^{\circ}$ & Station & Organisme & $\begin{array}{c}\text { Période } \\
\text { d'obser- } \\
\text { vation }\end{array}$ & $\begin{array}{l}\text { Période } \\
\text { utilisée }\end{array}$ \\
\hline 1 & Arles & DDE 13 & $1861-(x)$ & $1935-1972$ \\
2 & Salin de Giraud & $\left(^{*} 2\right)$ & $1874-(x)$ & $1935-1972$ \\
3 & Sambuc & MN & $(?)-(x)$ & $1935-1972$ \\
4 & Istres le Tubé & MN & $1921-(x)$ & $1931-1973$ \\
5 & Salon & MN & $1940-(x)$ & $1940-1973$ \\
6 & Avignon & $\left.1^{*} 3\right)$ & $1930-(x)$ & $1930-1973$ \\
7 & Avignon & $\left.1^{*} 4\right)$ & $1938-(x)$ & $1957-1973$ \\
8 & Graveson & EDF & $08 / 57-$ & $1958-1971$ \\
\hline
\end{tabular}

NB : $(x)$ Encore en service en 76.

(*2) Compagnie Salinière de Camargue

(*3) Campo Bello.

(*4) Usine des Eaux.

a) L'isotropie des lois statistiques est le premier point. Le graphique 3 a montré que cette isotropie est assez bien réalisée par saison pour les pluies de $1 \mathrm{~h}$. D'autres comparaisons graphiques confirment à peu près cette isotropie mais on a montré qu'elle n'est pas absolue. Le choix de la station longue durée est donc important et risque d'influer sur les résultats.

b) Le mélange des couples $\left(P, P_{M}\right)$ pour la même pluie nécessite comme première condition l'isotropie dont on vient de parler, mais en nécessite une deuxième qui est l'indépendance des observations. Cette deuxième condition est loin d'être réalisée ce qui risque de biaiser les résultats.

Par ailleurs, la méthode est sensible au mélange des observations, et (graphique 10) lorsqu'on ne les mélange pas, au choix du poste pluviométrique dont on tire la loi de la pluie ponctuelle. Il semble donc qu'on ait intérêt à ne conserver qu'un poste pluviométrique dans le calcul de la densité $\rho\left(P, P_{M}\right)$. Nous parlerons du choix de ce poste ci-après.

c) La taille de l'échantillon qui permet de calculer la densité $\rho$ est très insuffisante si elle n'est que de 4 années. Le graphique 10 montre par exemple l'écart obtenu par rapport à un échantillon de 20 ans. Il semble que la taille de l'échantillon ne doive pas être inférieure à 15 ans en climat contrasté pour être sûr des résultats.

d) La séparation en saisons, quoique décelable, semble introduire des différences inférieures à l'ordre de grandeur de l'incertitude sur le résultat. On peut donc la négliger. e) Le point le plus important concerne toutefois le calcul de la pluie moyenne et la définition de la pluie ponctuelle. On ne calcule pas en effet la pluie moyenne par la méthode des isohyètes qui serait trop compliquée à mettre en œuvre. On utilise donc la méthode de Thiessen, ce qui revient à affecter à chaque pluviomètre une zone d'influence déduite du quadrillage du réseau de mesures. Cela revient donc également à admettre que la pluie est constante sur la surface d'influence du pluviomètre. La pluie ponctuelle représente donc en fait la pluie moyenne sur une surface élémentaire, ce qui a pour conséquence que le mélange des observations n'est pas très valable : on ne mélange pas des pluies ponctuelles mais des pluies moyennes sur des surfaces différentes. Si on ne tient pas compte de cette caractéristique de la méthode de Thiessen on peut être conduit aux erreurs suivantes: lère erreur

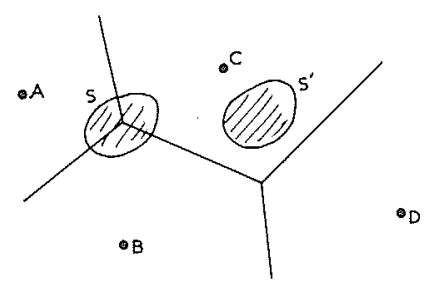

On calculerait un coefficient d'abattement sur la surface $S$ alors qu'on ne pourrait pas le calculer pour une surface $S^{\prime}$ identique mais située à l'intérieur de la zone d'influence du poste pluviométrique $C$. Ceci est en contradiction avec l'hypothèse d'isotropie.

2ème erreur :

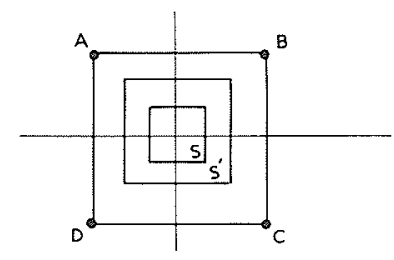

Pour deux surfaces différentes mais ayant la même proportion de chaque zone d'influence des pluviomètres, on obtiendrait le même coefficient d'abattement. Dans le cas du réseau de mesures carré, on voit immédiate: ment que $S, S^{\prime}$ et $\mathrm{ABCD}$ par exemple auront le même coefficient d'abattement.

\section{Synthèse}

Deux points se dégagent donc :

a) Le coefficient d'abattement sur une surface ne dépend pas de la position de cette surface (hypothèse d'isotropie). Il est donc illusoire par exemple de calculer un coefficient d'abattement sur un bassin versant en prenant une pluie moyenne égale à $S_{A} P_{A}+S_{B} P_{B}+$ $S_{C} P_{C}$. Cela ne signifie pas bien sûr que la meilleure estimation d'une pluie bien déterminée ne soit pas donnée par cette formule.

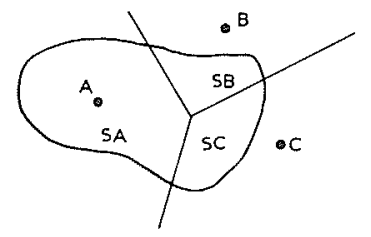


b) Il existe un seuil au-dessous duquel la structure du réseau pluviométrique ne permet pas d'obtenir le coefficient d'abattement. Comme il n'y a pas de gradient pluviométrique systématique, ce seuil est le plus petit des cercles centrés aux postes pluviométriques et inclus à l'intérieur des zones d'influences.

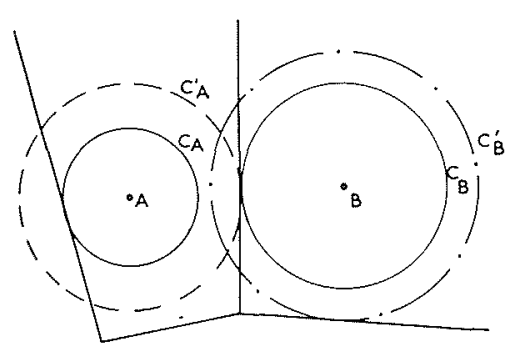

On peut donc calculer le coefficient d'abattement de la surface $C_{A}$ aux surfaces $C_{A}^{\prime}$ qui intéressent d'autres pluviomètres que $A$. Si on calcule le coefficient d'abattement de $C_{B}^{\prime}$ à $C_{B}$ on pourra se ramener à $C_{A}$ en le calculant sur une surface $C_{A}^{\prime}$ égale à $C_{B}$ et en faisant le produit des coefficients obtenus.

De toute façon on ne peut que calculer un coefficient d'abattement d'une pluie moyenne sur une surface (la plus petite étant $C_{A}$ ) à la pluie moyenne sur une autre surface. Le problème de l'abattement de la pluie ponctuelle à une pluie moyenne n'est donc pas résolu en toute rigueur. Pour cela, il faudrait restituer à la pluie obtenue en un poste pluviométrique sa qualité de pluie ponctuelle, donc calculer la pluie moyenne sur une surface de façon "dynamique", soit par la méthode des isohyètes, soit en simulant à partir du réseau existant les observations d'un réseau fictif suffisamment dense pour être utilisée de façon "statique".

\section{Resultats}

En appliquant la méthode expliquée ci-dessus (fig. 11), nous avons déterminé le cercle $G$, centré sur Graveson $\left(60 \mathrm{~km}^{2}\right)$ en-dessous duquel on ne peut calculer de coefficient d'abattement. Le seuil suivant $S_{1}$ $\left(177 \mathrm{~km}^{2}\right)$ est centré sur Sambuc. On cherche donc des coefficients d'abattement sur $G_{2}\left(177 \mathrm{~km}^{2}\right)$ puis $S_{2}\left(333 \mathrm{~km}^{2}\right)$ et $S_{3}\left(774 \mathrm{~km}^{2}\right)$. Pour $S_{2}$ et $S_{3}$, on a multiplié les résultats par ceux obtenus pour $G_{2}$. Les résultats obtenus sont présentés sur le graphique 12 . Cela demanderait confirmation, mais ces résultats sont sans doute valables pour la région méditerranéenne si l'influence du relief n'est pas prédominante.

\section{Rationalisation d'un réseau de mesures}

Signalons pour terminer que les considérations cidessus peuvent servir de point de départ à une réflexion sur la densité optimale d'un réseau de mesures et sur la taille minimale de la zone d'influence d'un poste pluviométrique en-dessous de laquelle l'imprécision de la mesure devient plus importante que la variation spatiale de la pluie. 